\title{
CRIANZA DE Eisenia foetida (LOMBRIZ ROJA) EN DIFERENTES SUSTRATOS DE DESARROLLO BIOLÓGICO
}

\section{BREEDING OF Eisenia foetida (RED WORM) IN DIFFERENT SUBSTRATES OF BIOLOGICAL DEVELOPMENT}

\author{
Angel Canales Gutiérrez ${ }^{1}$, Brayan Juan Solís Ramos², Rosmery Judith Panca Castañeda ${ }^{3}$ y \\ Bessy Laleska Quispe Cáceres ${ }^{4}$
}

\begin{abstract}
Resumen
El aprovechamiento de residuos orgánicos, es una alternativa ambiental viable, siendo las lombrices organismos que permiten un proceso rápido de descomposición. La investigación de crianza de lombrices rojas en diferentes sustratos de desarrollo biológico, se llevó a cabo durante junio y julio del 2019. Los objetivos fueron: a) Determinar la influencia del tipo de sustrato (tipo de tratamiento) en el incremento de cocones y biomasa de la lombriz roja, b) Estimar el grado de asociación entre número de lombrices, número de cocones, longitud y biomasa en diferentes tratamientos de sustrato. La metodología consistió en implementar tres tipos de sustrato y uno de control, considerando heces de animales y restos de frutas. La evaluación de la temperatura, humedad y $\mathrm{pH}$ fue cada dos días, y la del número de lombrices, cocones, longitud y biomasa fue cada 12 días. Los resultados fueron: el número de cocones de lombriz roja de tierra, se incrementó hasta seis cocones o huevos en 36 días (promedio 4.33 cocones/lombriz), siendo el tratamiento con estiércol de animales y cáscaras de fruta, donde hubo un mayor número de cocones, en relación a los otros tratamientos. El mayor grado de asociación se registró entre longitud ( $\mathrm{mm}$ ) y la biomasa (g) de lombrices, con un valor de $\mathrm{r}=0.82$.
\end{abstract}

Palabras clave: biomasa, crianza, heces, lombriz, residuos orgánicos, reproducción y sustrato.

\begin{abstract}
The use of organic waste is a viable environmental alternative, the sources being an organism that allows a rapid decomposition process. The research of rearing red worms in different substrates of biological development, was carried out during June and July 2019. The objectives were: a) To determine the influence of the type of substrate (type of treatment) on the increase of stumps and biomass of the red worm, b) Estimate the degree of association between number of worms, number of stumps, length and biomass in different substrate treatments. The methodology consists of implementing three types of substrate and one of control, analyzing animal faeces and fruit remains. Assessment of temperature, humidity and $\mathrm{pH}$ was performed every two days, and the number of earthworms, cocoons, length and biomass every 12 days. Results were as follows: the number of red earthworm cocoon increased to six cocoons or eggs in 36 days (average 4.33 cocoons/earthworm), with the treatment with animal manure and fruit peels, as the one with higher number of cocoons, in relation to the other treatments. The highest degree of association was recorded between length ( $\mathrm{mm}$ ) and the biomass $(\mathrm{g})$ of earthworms, with a value of $\mathrm{r}=0.82$.
\end{abstract}

Key words: biomass, breeding, faeces, worm, organic waste, reproduction and substrate.

\section{Introducción}

Las lombrices de tierra son animales que se agrupan en 13 familias, aunque se han descrito más de 5000 especies (Brown et al., 2013), la Eisenia foetida, es la más utilizada para el tratamiento de la lombricultura, pues es capaz de convertir casi cualquier tipo de desecho orgánico en un producto final denominado lombricomposta (Decaens et al., 2003). Estos abonos orgánicos mejoran tanto las propiedades químicas del suelo, como las propiedades físicas y biológicas (Martínez, 2009).

Una alternativa para mitigar la contaminación del ambiente, es el proceso de vermicompostaje (Ortiz \&
Fragoso., 2004), ya que puede ser usado como sustituto de los fertilizantes químicos (Khatua et al., 2018). Los residuos orgánicos podrían tener efectos beneficiosos, al generar proteína como parte de la alimentación animal (Fragoso \& Brown, 2007; Lalander et al., 2015), pudiendo utilizarse en forma fresca o molida como fuente aditiva de nutrientes (Chiripasi et al., 2013).

El vermicompost se obtiene por un proceso sencillo de descomposición (Khatua et al., 2018), bioquímica de materiales orgánicos (Fu et al., 2015; Zhu, 2000), que reduce el impacto ambiental evitando emisiones de gases de efecto invernadero (Hondupalma \& SNV, 2011). Además se pueden usar las excretas de los 
animales en conjunto con los desechos orgánicos y que pueden restaurar los suelos degradados (Diacono \& Montemurro, 2012).

Las lombrices, realizan una actividad de importancia en el medio ambiente (Oliveira et al., 2008), esta actividad consiste en la producción de abono orgánico (Durán \& Henríquez, 2009), ya que sus defecaciones constituyen un magnifico abono orgánico por el contenido de su flora bacteriana (Toccalino et al., 2004), teniendo la capacidad de alterar la materia orgánica y transformarla a nutrientes (Ávila et al. 2007) que se adhieren al suelo para una buena productividad (Mendoza, 2018).

La lombriz de tierra tiene una dinámica biológica en materia orgánica y en aguas residuales (Schuldt et al., 2005). Un buen sustrato para el crecimiento de lombrices son los residuos de café (Duraá \& Henríquez, 2009); sin embargo, al aprovechar los residuos de frutas, estos afectan su reproducción (Salinas et al., 2014) pero, con la mezcla de desechos leñosos se obtienen mejores resultados en población de lombrices (Yepes et al., 2008).

Para mejorar la eficiencia del proceso de reproducción de la lombriz de tierra se recomiendan muestras homogéneas sobre cada tratamiento (Aynehband et al., 2017), de igual manera es necesario realizar control, tratamientos y réplicas (Ávila et al., 2007) que puedan ser analizados mediante métodos estadísticos donde se compare con diferentes sustratos para la mezcla con biosólidos (Bahadori et al., 2017).

Los objetivos específicos fueron: a) Determinar la influencia del tipo de sustrato (tipo de tratamiento) en el incremento de cocones y biomasa de la lombriz roja, b) Estimar el grado de asociación entre número de lombrices, número de cocones, longitud y biomasa en diferentes tratamientos de sustrato.

\section{Materiales y Métodos}

Área de estudio

El estudio se realizó en el invernadero de la Universidad Nacional del Altiplano de Puno, ubicada a $3810 \mathrm{msnm}$ (Coordenadas 1549'42.94"S, $\left.70^{\circ} 0^{\prime} 58.90^{\prime \prime} \mathrm{O}\right)$, cuenta con un espacio para el experimento y registro de datos.

Frecuencia y horario de muestreo

Los registros del número de cocones y biomasa de las lombrices rojas, se realizaron durante 36 días (muestreo cada 12 días) en el horario de 09:00 a 10:00 h.

Los registros adicionales de temperatura $\left({ }^{\circ} \mathrm{C}\right)$, humedad (\%) y pH del suelo, se realizaron cada dos días durante 36 días en el horario de 09:00 a 15:00 h. Descripción del uso de materiales, equipos e insumos

Se utilizaron 200 lombrices de la especie Eisenia foetida, colocando en diez placas Petri veinte lombrices en cada una, para poder identificar a las lombrices más aptas para el estudio, tomando en cuenta su coloración y actividad, se descartaron las lombrices más débiles hasta llegar a los 100 individuos los cuales fueron colocados en los tratamientos para realizar la investigación.

Las celdas de crianza se construyeron con tablas de madera, con un espacio separado para cada tratamiento. Cada una de las celdas de tratamiento tuvo una dimensión de $0.50 \times 0.30 \times 0.15 \mathrm{~m}$, la preparación de los tratamientos se realizó de la siguiente manera:

- Tratamiento control (T0): tierra agrícola (100\%).

- Tratamiento 1 (T1): estiércol de vaca (40\%), estiércol de oveja (30\%), estiércol de caballo (30\%) y $2 \mathrm{~kg}$ de tierra agrícola.

- Tratamiento 2 (T2): cáscara de sandía (40\%), cáscara de plátano (30\%), cáscara de tuna (30\%) y $2 \mathrm{~kg}$ de tierra agrícola.

- Tratamiento 3 (T3): compuesto de T1 (50\%), T2 $(50 \%)$ y $2 \mathrm{~kg}$ de tierra agrícola (Tabla 1$).$

Para colocar el estiércol y las cáscaras de frutas se utilizó una balanza digital (VALTOX BRD10, capacidad de $5 \mathrm{~kg}$, dimensiones 116 × 230 × $246 \mathrm{~mm}$ ), luego las lombrices fueron distribuidas equitativamente en cada tratamiento (10 lombrices por tratamiento). Cada tratamiento tuvo dos repeticiones, mientras que el tratamiento control solo tuvo una repetición.

En cada tratamiento se colocó una tela polar con la finalidad de mantener la humedad y como sistema de aislamiento para que no puedan migrar las lombrices hacia los otros tratamientos. El riego para cada tratamiento fue cada 2 días, regando con $500 \mathrm{ml} \mathrm{de}$ agua.

Tabla 1. Composición porcentual de insumos utilizados para los sustratos de los tratamientos de crianza de lombrices. Realizado de junio a julio 2019.

\begin{tabular}{lcccc}
\hline $\begin{array}{l}\text { Tratamientos } \\
\text { materia orgánica }\end{array}$ & T0 & T1 & T2 & T3 \\
\hline Estiércol de vaca & $0 \%$ & $40 \%$ & $0 \%$ & $20 \%$ \\
$\begin{array}{l}\text { Estiércol de oveja } \\
\text { Estiércol de }\end{array}$ & $0 \%$ & $30 \%$ & $0 \%$ & $15 \%$ \\
caballo & & & $0 \%$ & $15 \%$ \\
Cáscara de sandia & $0 \%$ & $0 \%$ & $40 \%$ & $20 \%$ \\
Cáscara de tuna & $0 \%$ & $0 \%$ & $30 \%$ & $15 \%$ \\
Cáscara de plátano & $0 \%$ & $0 \%$ & $30 \%$ & $15 \%$ \\
\hline Total & $0 \%$ & $100 \%$ & $100 \%$ & $100 \%$
\end{tabular}

Cada 12 días se realizó el conteo del número de lombrices, número de cocones, biomasa y longitud. Para ello, la tierra de cada tratamiento se colocó sobre una mesa con la finalidad de registrar las variables consideradas en la investigación.

Para contar el número de lombrices y de cocones, se hizo mediante un conteo por observación; para el cálculo de biomasa $(\mathrm{g})$ y longitud $(\mathrm{mm})$ se tomaron tres individuos de cada tratamiento de manera aleatoria; la biomasa se calculó mediante una balanza analítica de precisión marca Sartorius, y la longitud se obtuvo utilizando una regla de $30 \mathrm{~cm}$.

Para evaluar el tipo de sustrato (monitoreos cada 2 días) con mayor énfasis se tomó en cuenta la 
temperatura $\left({ }^{\circ} \mathrm{C}\right)$, humedad $(\%)$ estos fueron obtenidos mediante un termohigrómetro (versión SH 110, rango de $10-99 \% \mathrm{RH})$, también se tomaron medidas de $\mathrm{pH}$ mediante el equipo $\mathrm{pH}$ - metro (Sensor de $\mathrm{pH}$ inalámbrico, PS - 3204) estos equipos fueron de la Oficina de Gestión Ambiental de la Universidad Nacional de Altiplano de Puno.

Variables analizadas

Variable independiente $=$ Tratamientos de sustrato

Variable dependiente $=$ Número de cocones $\mathrm{y}$ biomasa (g), número de lombrices, longitud (mm). $\underline{\text { Prueba estadística }}$

Se aplicó las pruebas estadísticas de Kruskal Wallis, ANDEVA y CORRELACIÓN, utilizando el Programa estadístico INFOSTAT 2018.

\section{Resultados y discusión}

Determinar la influencia del tipo de sustrato en el incremento de cocones y biomasa de la lombriz roja

El número de cocones o huevos de lombriz roja se incrementó hasta 6 cocones o huevos en 36 días (promedio 4.33 cocones), siendo el tratamiento 3 (50\% de estiércol de caballo, oveja, vaca; $50 \%$ cáscaras de tuna, sandía y plátano) donde hubo un mayor número de cocones en relación a los otros tratamientos (Figura 1). Asimismo, los promedios de $\mathrm{pH}$ en los tratamientos fueron similares $(\mathrm{T} 0=7.90 ; \mathrm{T} 1=8.57 ; \mathrm{T} 2=8.60 ; \mathrm{T} 3$ $=8.63)$, teniendo la tendencia de alcalinidad.

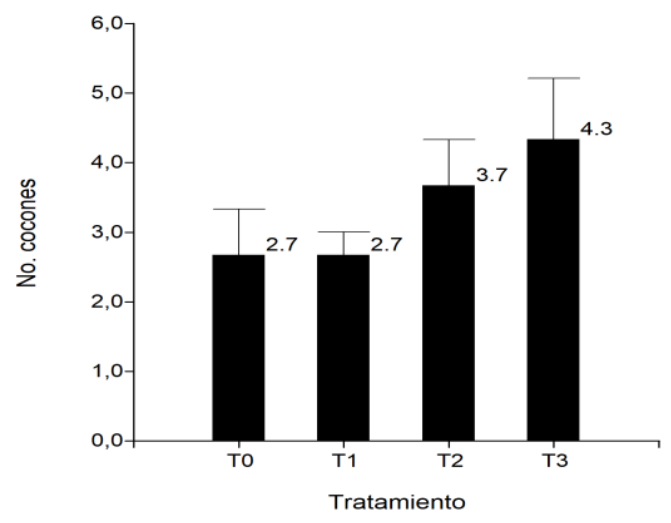

Figura 1. Análisis de comparación del número de cocones (huevos) de lombrices rojas criadas en diferentes tratamientos de sustrato de estiércol de ganado y cáscaras de frutas.

Sin embargo, no hubo diferencia estadística (Hcalc $(0.05)=3.65 ;$ G.L. $=3 ; \mathrm{P}=0.26)$. En el análisis determinamos que existió un mayor incremento de lombrices en el T3, el cual incrementó su población en $17 \%$ por mes (36 días), siendo un buen resultado en comparación con el trabajo realizado por Zhu (2000), quien obtuvo un incremento poblacional de 20\%; mientras que el nivel más bajo de incremento poblacional fue en T0 y T1 (con promedios de 2.67 en ambos casos), teniendo un incremento poblacional de $3 \%$ por mes (36 días), comparativamente con los resultados de Diacono \& Montemurro (2012); Lalander et al. (2015), están muy por debajo de media mínima $10 \%$, en el T3, hubo incremento de cocones, registrando un promedio de 3.67 cocones, mientras que la mayor producción se obtuvo en el T3, con un incremento de 4.3 cocones/mes (36 días), siendo este un registro similar de 3.5 cocones/mes (Ávila et al., 2007). Es probable que, en la producción de cocones, longitud y biomasa, hayan influido la temperatura, debido a que numéricamente fue mayor en el tratamiento 3 (Promedio $23.10{ }^{\circ} \mathrm{C}$ ), siendo menores en los otros tratamientos, sin embargo, los registros de humedad, fueron similares $(50,51,50 \%$ respectivamente), a excepción del T0, que fue de $54 \%$ (Tabla 2).

Tabla 2. Registro de temperatura y humedad durante los meses de investigación, sobre crianza de lombrices rojas.

\begin{tabular}{|c|c|c|c|c|c|c|c|}
\hline \multicolumn{4}{|c|}{ Temperatura $\left({ }^{\circ} \mathrm{C}\right)$} & \multicolumn{4}{|c|}{ Humedad (\%) } \\
\hline \multicolumn{4}{|c|}{ Tratamientos } & \multicolumn{4}{|c|}{ Tratamientos } \\
\hline T0 & $\mathrm{T} 1$ & $\mathrm{~T} 2$ & T3 & T0 & $\mathrm{T} 1$ & $\mathrm{~T} 2$ & $\mathrm{~T} 3$ \\
\hline 21.5 & 23.7 & 26.4 & 27.0 & 40 & 50 & 58 & 56 \\
\hline 23.9 & 21.2 & 21.3 & 20.8 & 59 & 55 & 56 & 59 \\
\hline 20.7 & 22.5 & 22.6 & 22.5 & 53 & 39 & 47 & 44 \\
\hline 23.5 & 23.5 & 18.1 & 23.4 & 42 & 51 & 58 & 49 \\
\hline 29.1 & 27.0 & 26.8 & 24.0 & 49 & 46 & 49 & 52 \\
\hline 20.0 & 20.1 & 20.5 & 20.4 & 68 & 46 & 52 & 54 \\
\hline 20.7 & 21.7 & 21.9 & 23.6 & 53 & 53 & 49 & 45 \\
\hline 23.5 & 21.3 & 23.1 & 23.8 & 42 & 53 & 41 & 52 \\
\hline 17.0 & 20.8 & 21.3 & 19.9 & 58 & 48 & 48 & 47 \\
\hline 23.9 & 20.0 & 22.4 & 23.7 & 59 & 54 & 51 & 49 \\
\hline 29.1 & 28.1 & 28.4 & 29.1 & 49 & 49 & 50 & 47 \\
\hline 18.9 & 20.3 & 20.3 & 20.0 & 68 & 55 & 55 & 56 \\
\hline 23.9 & 20.8 & 21.3 & 22.2 & 59 & 48 & 53 & 45 \\
\hline 21.5 & 20.6 & 25.2 & 26.8 & 40 & 54 & 53 & 50 \\
\hline 29.1 & 21.7 & 23.5 & 20.6 & 49 & 49 & 48 & 47 \\
\hline 20.0 & 20.9 & 22.5 & 22.5 & 68 & 52 & 51 & 49 \\
\hline 25.0 & 28.1 & 20.9 & 25.7 & 49 & 46 & 50 & 47 \\
\hline 17.0 & 20.3 & 22.8 & 20.0 & 68 & 55 & 55 & 56 \\
\hline 22.7 & 22.4 & 22.7 & 23.1 & 54 & 50 & 51 & 50 \\
\hline
\end{tabular}

Se tuvieron registros más bajos de producción de cocones en $\mathrm{T} 0$ y T1, presentando un incremento de 2.67 cocones / mes (36 días), siendo similares con los registros que obtuvo de 3.0 cocones/mes (Mendoza, 2018).g

La biomasa ( $\mathrm{g}$ ) de lombrices criadas en 36 días fue mayor en $\mathrm{T} 3$, registrando $0.6 \mathrm{~g}$, siendo un poco menor en $\mathrm{T} 0=0.51 ; \mathrm{T} 1=0.57 ; \mathrm{T} 2=0.58$ (Figura 2$)$.

La biomasa ( $\mathrm{g}$ ) de las lombrices rojas, criadas en diferentes tratamientos de sustrato, presentan diferencias, siendo mayor la biomasa en el T3 (0.61) y menores en los otros tratamientos (Fcalc $(0.05)=4.05$; G.L. $=3 ; \mathrm{P}=0.051)$. 


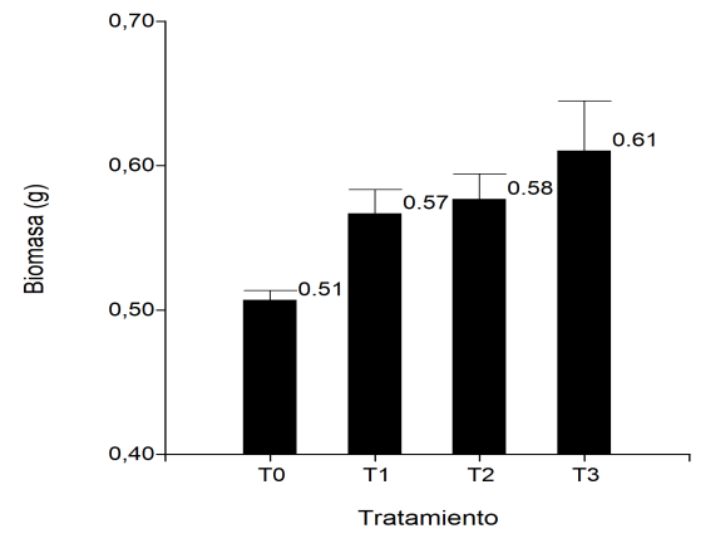

Figura 2. Análisis comparativo de la biomasa $(\mathrm{g}) \mathrm{de}$ lombrices criadas en diferentes tratamientos de sustrato de estiércol de ganado y cáscaras de frutas.

El tipo de alimento que ingieren las lombrices influye en su crecimiento y desarrollo (Diacono \& Montemurro, 2012), influyendo en el incremento de la biomasa y presenten un comportamiento más activo. Las lombrices que estuvieron en el T3, tuvieron una mejor capacidad reproductiva en sustratos con estiércol de animales y cáscaras de fruta. Asimismo, la materia orgánica del compostaje presenta una alta acumulación de nutrientes de N, P, K y Ca (Jiménez et al., 2019) y está característica fue importante para el desarrollo de la investigación. Por ejemplo, el estiércol de caballo posee alto $\mathrm{N}$ y de característica salina (Morales et al., 2009), adicionalmente, la cáscara de tuna permite mantener la humedad del sustrato y adicionando vitamina C (Terán et al., 2015), en la investigación la humedad estuvo alrededor de 50\%, asimismo, la cáscara del plátano ha aportado al sustrato $\mathrm{Fe}, \mathrm{Zn}, \mathrm{K}$, $\mathrm{Ca}, \mathrm{Cu}$ y Mn (Martínez et al., 2016), finalmente, el estiércol de ganado vacuno, es fuente de nutrición debido a que presenta $\mathrm{N}, \mathrm{P}, \mathrm{K}$ y un alto contenido de micronutrientes y libre de agentes (Peralta et al., 2016). Estas condiciones de mezcla de estiércol ganado y cáscaras de fruta, fue importante para la crianza de la lombriz roja, que pueden utilizarse para diferentes usos de producción orgánica.

Estimar el grado de asociación entre número de lombrices, número de cocones, longitud y biomasa en diferentes tratamientos de sustrato.

El grado de asociación entre las variables dependientes de: número de lombrices, número de cocones, longitud de lombrices y biomasa, se comportaron con una afinidad o asociación entre un rango de $r=0.63$ hasta $r=0.82$. La mejor asociación presenta las variables longitud de lombrices $(\mathrm{mm})$ y biomasa $(\mathrm{g})$, siendo un $\mathrm{r}=0.82$ (Tabla 3 ).

Las variables analizadas tuvieron influencia del uso del estiércol de animales y restos de frutas, que tienen influencias en el crecimiento y desarrollo de la lombriz, donde se muestra que las lombrices en un sustrato con estiércol presentan una mayor biomasa (Ávila et al., 2007; Durán \& Henriquez, 2009; Mendoza, 2018), al igual que en nuestra investigación haciendo que estas obtengan una mayor biomasa $(0.63 \mathrm{~g})$ y longitud corporal $(74.5 \mathrm{~mm})$ con el tratamiento donde se consideró estiércol de vaca, oveja y caballo, incluyendo cáscaras de fruta como plátano, sandía y tuna. Sin embargo, fueron menores las longitudes en los T0, T1, T2, que registraron $50.5 ; 73.7 ; 73.9 \mathrm{~mm}$ respectivamente.

Tabla 3. Grados de asociación de variables dependientes que influyen en la crianza de lombrices en diferentes tratamientos de sustrato.

\begin{tabular}{|c|c|c|c|}
\hline 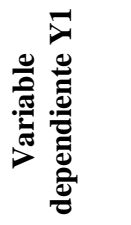 & 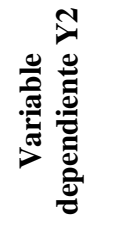 & 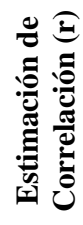 & 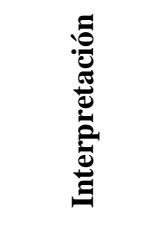 \\
\hline $\begin{array}{l}\text { Nro. } \\
\text { lombrices }\end{array}$ & $\begin{array}{l}\text { Nro. } \\
\text { cocones }\end{array}$ & 0.63 & $\begin{array}{l}63 \% \mathrm{de} \\
\text { asociación }\end{array}$ \\
\hline $\begin{array}{l}\text { Longitud } \\
\text { de } \\
\text { lombrices } \\
(\mathrm{ml})\end{array}$ & $\begin{array}{l}\text { Nro. } \\
\text { cocones }\end{array}$ & 0.76 & $\begin{array}{l}76 \% \text { de } \\
\text { asociación }\end{array}$ \\
\hline $\begin{array}{l}\text { Longitud } \\
\text { de } \\
\text { lombrices } \\
(\mathrm{ml})\end{array}$ & $\begin{array}{l}\text { Biomasa } \\
(\mathrm{g})\end{array}$ & 0.82 & $\begin{array}{l}82 \% \text { de } \\
\text { asociación }\end{array}$ \\
\hline
\end{tabular}

Asimismo, la asociación entre temperatura humedad, es muy importante para que las lombrices puedan desarrollarse adecuadamente, ya que si la humedad está por debajo del $60 \%$, las lombrices rojas, empiezan a sufrir estrés, influyendo a que las lombrices bajen su ritmo de alimentación y, por ende, presentarían una menor capacidad de reproducirse (Mendoza, 2018).

\section{Conclusiones}

El tratamiento que incluyó $20 \%$ de estiércol de vaca, $15 \%$ estiércol de oveja, $15 \%$ estiércol de caballo, $20 \%$ de cáscara de sandía, $15 \%$ cáscara de tuna y $15 \%$ cáscara de plátano, fue el más adecuado para el incremento de cocones y biomasa.

Se ha incrementado hasta 6 cocones en 36 días y una biomasa de $0.67 \mathrm{~g}$, logrando un incremento de $21.8 \%$ en 36 días.

Existe un grado de asociación de $\mathrm{r}=0.82$ entre las variables dependientes de longitud de lombrices ( $\mathrm{mm}$ ) y biomasa $(\mathrm{g})$.

\section{Agradecimientos}

A la Oficina de Gestión Ambiental de la Universidad Nacional del Altiplano de Puno, por el apoyo de equipos y acceso al invernadero. 


\section{Literatura citada}

Ávila G., Hernán G., Mario M. \& Alexander N. 2007. Reproduction of Eisenia foetida in agricultural soils from mining areas contaminated with copper and arsenic. Pesquisa Agropecuária Brasileira, 42: 435-441. DOI: http://dx.doi.org/10.1590/S0100-204X2007000300018.

Aynehband A., Aram G. \& Abdol A. 2017. Vermicompost: an eco-friendly technology for crop residue management in organic agriculture. Energy Procedia, 141: 667-671. DOI: https://doi.org/10.1016/j.egypro.2017.11.090.

Bahadori Z., Esmaielzadeh L., Karimi-torshizi M.A., Seidavi A., Olivares J., Hernández S., Salem A.Z.M. \& López S. 2017. The effect of earthworm (Eisenia foetida) meal with vermi-humus on growth performance, hematology, immunity, intestinal microbiota, carcass characteristics, and meat quality of broiler chickens. Livestock Science, 202: 74-81.

DOI: https://doi.org/10.1016/j.livsci.2017.05.010.

Brown G.G., Callaham M.A., Niva C.C., Feijoo A., Sautter Klaus D., James S.W., Fragoso C., Pasini A. \& Schmelz R.M. 2013. Terrestrial oligochaete research in Latin America: the importance of the Latin American meetings on oligochaete ecology and taxonomy. Applied Soil $\begin{array}{llll}\text { Ecology, } & 69: & 2-12 . & \text { DOI: }\end{array}$ https://doi.org/10.1016/j.apsoil.2012.12.006.

Chiripasi S.C., Moreki J.C., Nsoso S.J. \& Letso M. 2013. Effect of feeding mopane worm meal on mineral intake, retention and utilization in guinea fowl under intensive system. International Journal of Poultry Science, 12(1): 19-28. DOI: https://dx.doi.org/10.3923/ijps.2013.19.28.

Decaens T., Mariani L. \& Lavelle P.M. 2003. Las Comunidades de la macrofauna de la superficie del suelo asociadas con las heces de las lombrices de tierra en los llanos orientales de Colombia. 285-305. En: Jiménez Jaén, J.J. \& Thomas, R.J. (editores.) El arado natural: Las comunidades de macroinvertebrados del suelo en las sabanas neotropicales de Colombia. Centro Internacional de Agricultura Trópica.

Diacono M. \& Montemurro F. 2012. Long-term effects of organic amendments on soil fertility. Agronomy for Sustainable Development, 30: 401-422. DOI: https://doi.org/10.1051/agro/2009040.

Durán L. \& Henríquez C. 2009. Crecimiento y reproducción de la lombriz roja (Eisenia foetida) en cinco sustratos orgánicos. Agronomía Costarricense, 33 (2): 275-281. https://www.redalyc.org/articulo.oa?id=43613279011.

Fragoso C. \& Brown C.C. 2007. Ecología y taxonomía de las lombrices de tierra en Latinoamérica: el primer encuentro latino-americano de ecología y taxonomía de oligoquetos (ELAETAO1). 33-75. In: Brown G.G. \& Fragoso C. (eds.) Minhocas na América Latina: Biodiversidade e Ecologia. EMBRAPA Soja. Londrina. https://www.alice.cnptia.embrapa.br/handle/doc/469788.

Fu X., Huang K., Chen X., Li F. \& Cui G. 2015. Feasibility of vermistabilization for fresh pelletized dewatered sludge with earthworms bimastus parvus. Bioresource Technology, 175: 646-650. DOI: https://doi.org/10.1016/j.biortech.2014.11.007
Jiménez M.M., Gómez R., Oliva J., Granados L., PatFernández J.M. \& Aranda E.M. 2019. Influencia del estiércol composteado y micorriza arbuscular sobre la composición química del suelo y el rendimiento productivo de maíz forrajero (Zea mays L.). Nova Scientia, 11(23): $165 . \quad$ DOI: https://doi.org/10.21640/ns.v11i23.1957.

Hondupalma \& SNV. 2011. Manejo de residuos sólidos. Una Guía Para Socios y Personal de Hondupalma. https://www.academia.edu/15179746/Manejo_de_residu os_s\%C3\%B3lidos_Una_gu\%C3\%ADa_para_socios_y _personal_de_HONDUPALMA.

Khatua C., Sengunpta S., Balla V.K., Kundu B., Chakraborti A. \& Tripathi S. 2018. Dynamics of organic matter decomposition during vermicomposting of banana Stem waste using Eisenia foetida. Waste Management, 79: 287-295. DOI: https://doi.org/10.1016/j.wasman.2018.07.043.

Lalander C.H., Komakech A.J. \& Björn V. 2015. Vermicomposting as manure management strategy for urban small-holder animal farms - Kampala Case Study. Waste Management, 39: 96-103. DOI: https://doi.org/10.1016/j.wasman.2015.02.009.

Martínez C. 2009. Abonos orgánicos: lombricultura. En: Martínez C. (editor.) Sistema de agronegocios de Traspatio. México, Secretaría de Agricultura, Ganadería, Desarrollo rural, Pesca y Alimentación (SAGARPA). https://www.yumpu.com/es/document/read/14266676/lo mbricultura-sagarpa.

Martínez C., Cayón G., \& Ligarreto G. 2016. Composición química y distribución de materia seca del fruto en genotipos de plátano y banano. Corpoica Cienc Tecnol Agropecuaria, 17(2): 217-227. DOI: https://doi.org/10.21930/rcta.vol17_num2_art:491.

Mendoza E.L. 2018. Efecto de tres dosis de sustratos en la alimentación de la lombriz roja californiana (Eisenia foétida) con estiércol bovino y aserrín descompuesto en Sapecho, Alto Beni. Revista APTHAPI, 4:1128-1138. http://ojs.agro.umsa.bo/index.php/ATP/article/view/246.

Terán Y., Navas D., Petit D., Garrido E. \& D'Aubeterre R. 2015. Análisis de las características físico--químicas del fruto de Opuntia ficus-indica, cosechados en Lara, Venezuela. Revista Iberoamericana de Tecnología Postcosecha, 16(1): 69-74. https://www.redalyc.org/articulo.oa?id=81339864010.

Morales J.C., Fernández M.V., Montiel A. \& Peralta B.C. 2009. Evaluación de sustratos orgánicos en la producción de lombricomposta y el desarrollo de lombriz (Eisenia foetida). Biotecnia, 11(1): 19-26. https://biotecnia.unison.mx/index.php/biotecnia/article/v iew/49.

Peralta L., Juscamaita J. \& Meza V. 2016. Obtención y caracterización de abono orgánico líquido a través del tratamiento de excretas del ganado vacuno de un establo lechero usando un consorcio microbiano ácido láctico. Ecol. apl., 15(1): 1-10. DOI: http://dx.doi.org/10.21704/rea.v15i1.577.

Oliveira E.M., Costa F.J. \& Costa C.C. 2008. Reproduçao de minhoca (Eisenia foetida) em diferentes substratos. Revista Caatinga, 21: 146-150. https://www.redalyc.org/pdf/2371/237117585021.pdf. 
Ortiz A.I. \& Fragoso C. 2004. Earthworm populations under tropical maize cultivation: the effect of mulching with velvetbean. Biology and Fertility of Soils, 39: 438-445. DOI: https://doi.org/10.1007/s00374-004-0732-8.

Salinas F., Sepúlveda L. \& Sepulveda-Chavera G. 2014. Evaluación de La calidad química del humus de lombriz roja californiana (Eisenia foetida) elaborado a partir de cuatro sustratos orgánicos en Arica. Idesia, 32: 95-99. DOI: http://dx.doi.org/10.4067/S071834292014000200013.

Schuldt M., Rumi A. \& Gutiérrez D.E. 2005. Determinación de "edades" (clases) en poblaciones de E. foetida (Annelida: Lumbricidae) y sus implicancias reprobiológicas. Revista del Museo de la Plata Zoología, 17: $1-10$.

https://www.fcnym.unlp.edu.ar/uploads/docs/rmlp_zoo_ 2005_t17_n170.pdf.
Toccalino P.A., Agüero M.C., Serebrinsky C.A. \& Roux J.P. 2004. Comportamiento reproductivo de lombriz roja californiana (Eisenia foetida) según estación del año y tipo de alimentación. Revista Veterinaria, 15: 65-69. https://revistas.unne.edu.ar/index.php/vet/article/view/20 02.

Yepes S.M., Montoya L.J. \& Orozco F. 2008. Valorización de residuos agroindustriales - frutas - en Medellín y el sur del valle del Aburrá, Colombia. Rev.Fac.Nal.Agr.Medellìn, 61: 4422-4431. https://revistas.unal.edu.co/index.php/refame/article/vie $\mathrm{w} / 24742 / 25303$.

Zhu J. 2000. A review of microbiology in swine manure odor control. Agriculture, Ecosystems and Environment, 78: 93-106. DOI: https://doi.org/10.1016/S01678809(99)00116-4.

\footnotetext{
${ }^{1}$ Facultad de Ciencias Biológicas (Programa de Ecología) / Universidad Nacional del Altiplano de Puno / Perú. acanales@unap.edu.pe. https://orcid.org/0000-0002-3096-1705.

2 Instituto de investigación CHARLES DARWIN / Facultad de Ciencias Biológicas (Programa de Ecología) / Universidad Nacional del Altiplano de Puno / Perú.72197727@pronabec.edu.pe.

${ }^{3}$ Instituto de investigación CHARLES DARWIN / Facultad de Ciencias Biológicas (Programa de Ecología) / Universidad Nacional del Altiplano de Puno / Perú. rosmeryjudith15@gmail.com.

${ }^{4}$ Instituto de investigación CHARLES DARWIN / Facultad de Ciencias / Universidad Nacional del Altiplano de Puno / Perú. bessylaleska@gmail.com.
} 